\title{
The radial alveolar count method of Emery and Mithal: a reappraisal 2-Intrauterine and early postnatal lung growth
}

\author{
THOMAS P COONEY, WILLIAM M THURLBECK \\ From the Department of Pathology, University of British Columbia, Vancouver, Canada
}

\begin{abstract}
The radial count method of Emery and Mithal was applied to the lungs of 37 infants of gestational age 19-42 weeks. The method could be used satisfactorily to evaluate airspacecontaining lungs, whether alveolated or non-alveolated (saccule-containing). There was a progressive increase in complexity of terminal lung units throughout gestation, and a smooth transition was effected at 1 month of age between the radial counts of the intrauterine cohort and those of a separate group used to study postnatal lung growth. In the intrauterine and early postnatal group radial count results correlated very closely with the total gestational age (gestational age plus survival time after birth) of the child $(r=+0.93)$. Prior inflation of the lungs affected the radial counts of alveolated lungs much more than those of saccule-containing lungs, so that results correlated most closely with those of Emery and Mithal in the period up to 34 weeks' gestation. Radial count estimation correlated better with total gestational age, crown-rump length, body weight, and fixed lung volume than did any other morphometric parameter assessed. The radial count method provides a reliable index of lung growth in intrauterine and early postnatal development.
\end{abstract}

The radial alveolar count method of Emery and Mithal ${ }^{1}$ was introduced to assess the complexity of the terminal lung unit at all stages of development from 24 weeks' gestation to $12 \cdot 5$ years of age. In our previous paper (pp 572-9) we report an examination of the method and then the application of this technique in the postnatal period. This study examines the use of the method in the intrauterine and early postnatal period and relates the results to those of the postnatal cohort.

In applying the method to intrauterine lung growth we have specifically investigated the following problems: (1) Identification of terminal lung units before alveolarisation; (2) application of the method to non-alveolated terminal lung units-that is, assessment of the canalicular and terminal sac periods of development; (3) examination of the

Address for reprint requests: Dr WM Thurlbeck, Department of Pathology, University of British Columbia Health Sciences Centre Hospital, 2211 Wesbrook Mall, Vancouver, BC V6T 1W5, Canada. complexity of these units with increasing gestational age; (4) assessment of the effect of inflation before fixation on the radial counts during intrauterine and early postnatal lung growth.

\section{Methods}

Lungs from 37 infants of gestational age $19-42$ weeks were examined (18 male and 19 female). Eleven infants were stillborn, and the survival time of the 26 liveborn children ranged from 1 hour to 28 days. As in the complementary study of normal postnatal lung growth, the cases were selected to illustrate lung growth during normal pregnancy, $59 \%$ of the infants having died as a result of obstetric accidents or acute infections and the remainder having been premature or stillborn infants in whom no pathological abnormality was identified. All cases of congenital anomaly or chronic disease were excluded from the study. All cases had complete morphometric evaluation and results were available for comparison with radial counts. ${ }^{2}$ 


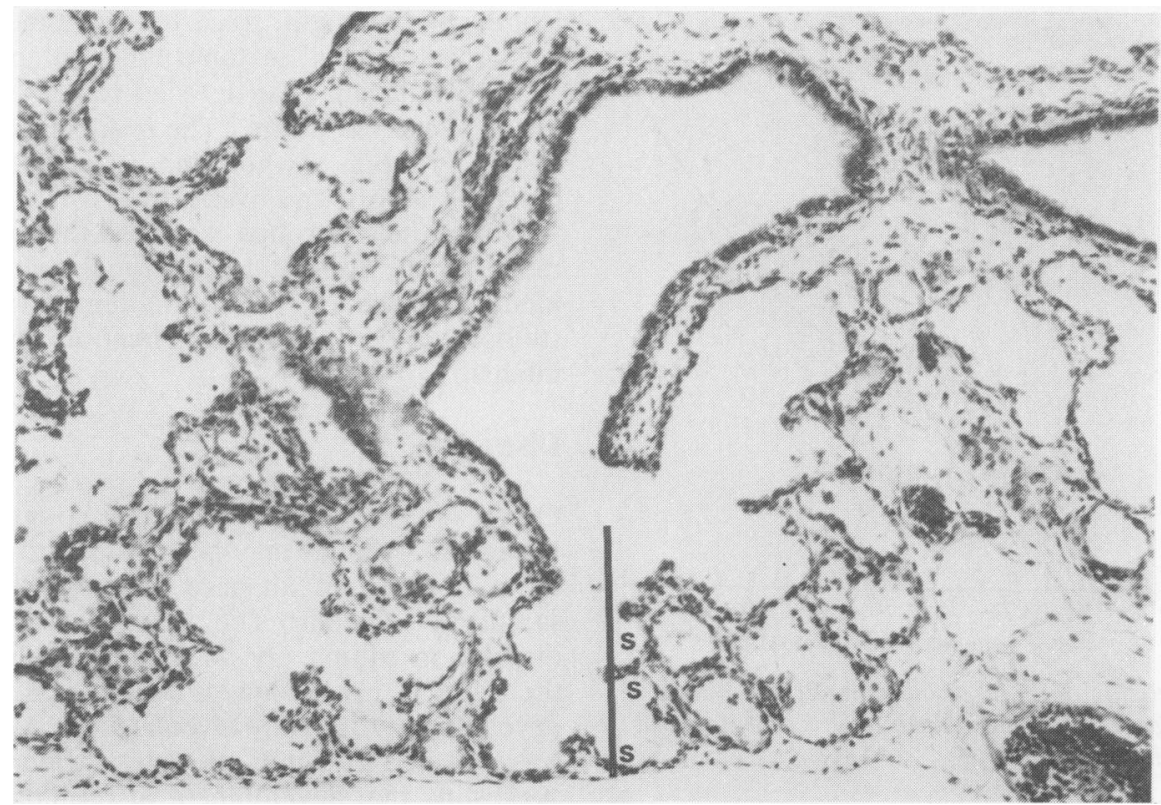

Fig 1 Radial count estimation in a 23-week fetus. The perpendicular is placed from the junction of conducting and respiratory epithelia to the nearest connective tissue septum. Saccules $(S)$ are counted. (Haematoxylin and eosin, $\times 40$ )

Inflation and sampling of lungs was according to the established protocol for this laboratory. ${ }^{3}$ Details of the application of the radial count method have been documented in the study of postnatal lung growth. When the lungs contained true alveoli the method was applied as described. Evaluation of non-alveolated but airspace-containing lungs necessitated the following modifications. (1) Lungs in the embryonal or pseudoglandular periods of development were by definition excluded from the study (no true airspaces). (2) Lungs containing large (canalicular period) or medium-sized (terminal sac period) saccules were deemed suitable for evaluation, and counts were made of the primitive airspaces (fig 1). Each distinct airspace was given a count of 1 , irrespective of whether the lung was in the canalicular or the terminal sac period. (3) Terminal lung units were defined as those portions of the parenchyma distal to the last strictly conducting airway. The most proximal part of the terminal lung unit (corresponding to the respiratory bronchiole in alveolated lungs) was identified as the junction between conducting epithelium and respiratory epithelium (fig 1). In the more immature lungs respiratory bronchioles were not identifiable, but the junction of conducting and respiratory epithelia was found to be an easily recognisable and constant landmark. (4) A perpendicular was placed from the junction of conducting and respiratory epithelia to the nearest connective tissue septum or pleura, and all airspaces (saccules) traversed by the line were counted (fig 1). Counts were made only in fields where a definitive morphological relationship of "respiratory" bronchiole to connective tissue septum or pleura could be discerned-that is, only definitive terminal lung units were chosen.

The age and sex of the patient were withheld from the observer when the counts were made. Suitable fields were very numerous in the more immature lungs: in keeping with the reproducibility data derived from the study of postnatal lungs a maximum of 40 counts per case was assessed, all available sections being used equally. In the whole group this resulted in the evaluation of a mean of 38 fields per case distributed through a mean of eight sections per case.

Radial count data for the whole group were correlated with total gestational age (that is, actual gestational age plus survival age), crown-rump length, body weight, bone age, and fixed lung volume. The correlation of radial counts with total gestational age was also examined for the subgroups stillborn/ liveborn and male/female.

Statistical evaluation of the results was by means of correlation coefficient (r) and Student's paired $t$ test. 


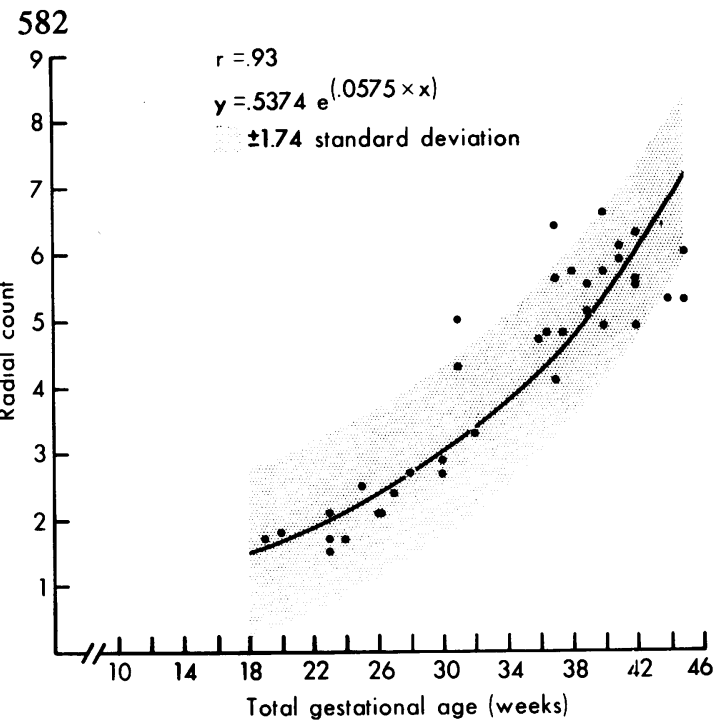

Fig 2 Correlation of radial count estimation with total gestational age. The exponential curve fit \pm 1.74 standard deviation is shown.

\section{Results}

Figure 2 depicts the correlation of the radial count estimation with the total gestational age of each patient. The exponential curve fit together with \pm 1.74 standard deviations is shown. A highly significant correlation was found $(r=+0.93)$. A similarly significant correlation was found when the radial count results were compared with crownrump length, body weight, and fixed lung volume; but the correlation with bone age was less exact (table 1).

The correlations of various morphometric parameters with total gestational age, crown-rump length, body weight, fixed lung volume, and bone age are also listed in table 1 for comparison with radial count data. The detailed results of this study are provided elsewhere. ${ }^{2}$ The results listed are those for the group as a whole (all cases, both male and female, stillborn and liveborn).

Within the subgroups examined the correlation of radial count with total gestational age was almost identical for male $(r=+0.91)$, female $(r=+0.93)$, stillborn $(r=+0.93)$, and liveborn $(r=+0.92)$ infants.

\section{Discussion}

Our results show that the radial count method of Emery and Mithal may be satisfactorily applied in the estimation of airspace development, whether saccular or alveolar. The data reveal a progressive increase in complexity of terminal lung units from the initial establishment of airspaces to the development of mature alveoli (fig 2). No difference was detected in counts of male and female infants, suggesting that development of terminal lung units proceeds at a similar rate in both sexes.

Correlation of radial count estimations with total gestational age, crown-rump length, body weight, fixed lung volume, and bone age shows highly significant correlation coefficients (table 1). Comparison with results derived from point counting, intercept counting, measurement of wall thickness, and alveolar counting (table 1) showed that the radial count estimation correlated better with gestational age, crown-rump length, body weight, and fixed lung volume than did the other morphometric variables, with the exception of internal surface area, the calculation of which includes multiple variables.

Table 1 Correlation coefficients of various morphometric parameters* with total gestational age ${ }^{* *}$, crown-rump length, body weight, fixed lung volume, and bone

\begin{tabular}{|c|c|c|c|c|c|}
\hline $\begin{array}{l}\text { Morphometric } \\
\text { parameters }\end{array}$ & $\begin{array}{l}\text { Total gestational } \\
\text { age }\end{array}$ & $\begin{array}{l}\text { Crown-rump } \\
\text { length }\end{array}$ & Body weight & $\begin{array}{l}\text { Fixed lung } \\
\text { volume }\end{array}$ & Bone age \\
\hline $\begin{array}{l}\text { Radial count } \\
\text { Volume proportion alveoli } \\
\text { Volume proportion alveolar ducts } \\
\text { Volume proportion non-parenchyma } \\
\text { Mean wall thickness } \\
\text { Mean airspace diameter } \\
\text { Internal surface area } \\
\text { Alveoli per unit area }\left(/ \mathrm{cm}^{2}\right) \\
\text { Alveoli per unit volume }(/ \mathrm{ml}) \\
\text { Total alveolar number (millions) }\end{array}$ & $\begin{array}{l}+0.93 \\
+0.69 \\
+0.66 \\
-0.15 \\
-0.82 \\
+0.61 \\
+0.95 \\
+0.59 \\
+0.57 \\
+0.70\end{array}$ & $\begin{array}{l}+0.90 \\
+0.69 \\
+0.60 \\
+0.08 \\
-0.78 \\
+0.54 \\
+0.93 \\
+0.62 \\
-0.62 \\
+0.72\end{array}$ & $\begin{array}{l}+0.89 \\
+0.66 \\
+0.62 \\
-0.10 \\
-0.77 \\
+0.54 \\
+0.91 \\
+0.53 \\
+0.53 \\
+0.65\end{array}$ & $\begin{array}{l}+0.89 \\
+0.63 \\
+0.72 \\
-0.18 \\
-0.79 \\
+0.69 \\
+0.95 \\
+0.40 \\
+0.36 \\
+0.56\end{array}$ & $\begin{array}{l}+0.66 \\
+0.65 \\
+0.46 \\
-0.03 \\
-0.77 \\
+0.43 \\
+0.74 \\
+0.50 \\
+0.51 \\
+0.50\end{array}$ \\
\hline
\end{tabular}

* See reference 2 for detailed data.

${ }^{* *}$ Total gestational age $=$ gestational age + survival age (best fit given in each instance).

$\begin{aligned} & \mathbf{r}>+0.52 \\ & \mathbf{r}<-0.52\end{aligned} \quad \mathrm{p}<0.001$

$$
\begin{array}{lll}
r>+0.42 & p<0.01 & r>+0.325 \\
r<-0.42 & & p<0.05 \\
& & r<-0.325
\end{array}
$$


Table 2 Predicted radial count values for various ages, derived from the intrauterine and postnatal cohorts

\begin{tabular}{|c|c|c|c|}
\hline $\begin{array}{l}\text { Gestation } \\
\text { (w) }\end{array}$ & $\begin{array}{l}\text { Radial } \\
\text { count }\end{array}$ & $\begin{array}{l}\text { Months } \\
\text { after birth }\end{array}$ & $\begin{array}{l}\text { Radial } \\
\text { count }\end{array}$ \\
\hline $\begin{array}{l}18 \\
19 \\
20 \\
21 \\
22 \\
23 \\
24 \\
25 \\
26 \\
27 \\
28 \\
29 \\
30 \\
31 \\
32 \\
33 \\
34 \\
35 \\
36 \\
37 \\
38 \\
39 \\
40 \\
41 \\
42 \\
43 \\
44\end{array}$ & $\begin{array}{l}1 \cdot 5 \\
1 \cdot 6 \\
1 \cdot 7 \\
1 \cdot 8 \\
1 \cdot 9 \\
2 \cdot 0 \\
2 \cdot 1 \\
2 \cdot 3 \\
2 \cdot 4 \\
2 \cdot 5 \\
2 \cdot 7 \\
2 \cdot 8 \\
3 \cdot 0 \\
3 \cdot 2 \\
3 \cdot 4 \\
3 \cdot 6 \\
3 \cdot 8 \\
4 \cdot 0 \\
4 \cdot 3 \\
4 \cdot 5 \\
4 \cdot 8 \\
5 \cdot 1 \\
5 \cdot 4 \\
5 \cdot 7 \\
6 \cdot 0 \\
6 \cdot 4 \\
6 \cdot 8(6 \cdot 75)^{*}\end{array}$ & $\begin{array}{r}1 \\
2 \\
3 \\
4 \\
5 \\
6 \\
8 \\
10 \\
20 \\
30 \\
40 \\
50 \\
60 \\
70 \\
80 \\
90 \\
100 \\
110 \\
120 \\
130 \\
140 \\
150 \\
160 \\
170 \\
180 \\
190 \\
200\end{array}$ & $\begin{array}{l}6.7 \\
7.4 \\
7 \cdot 8 \\
8 \cdot 1 \\
8 \cdot 3 \\
8 \cdot 5 \\
8 \cdot 8 \\
9 \cdot 0 \\
9.7 \\
10 \cdot 1 \\
10 \cdot 4 \\
10 \cdot 6 \\
10 \cdot 8 \\
11 \cdot 0 \\
11 \cdot 1 \\
11.2 \\
11 \cdot 3 \\
11.4 \\
11 \cdot 5 \\
11.6 \\
11.7 \\
11.7 \\
11.8 \\
11.9 \\
11.9 \\
12.0 \\
12.0\end{array}$ \\
\hline
\end{tabular}

${ }^{*}$ Results corrected to one decimal place.

The data from the present study were correlated with those from the study of postnatal lung growth. The point at which the groups met (44 weeks' gestation, 1 month of age) showed identical radial counts as predicted from 'the best fit of results in each group (radial count at 44 weeks' gestation $=$ 6.75 ; radial count at 1 month of age $=6 \cdot 7$ ). Predicted radial counts for various ages derived from the intrauterine and postnatal groups are listed in table 2 .

The results of this study were compared with those of Emery and Mithal (see fig 7 of the paper on postnatal lung growth-p 578). At all ages our radial counts were higher, but the difference was less before than after 34 weeks' gestation. It was at 32 weeks' gestation that alveoli were first recognisable in these lungs. ${ }^{2}$ Possibly the effect of inflation on radial count estimation is less important before 34 weeks, when the existing air spaces are relatively constant in appearance owing both to the large amount of interstitial tissue and to the intrinsic simplicity of the airspaces.

Comparison of the results of radial count estimations in the intrauterine and postnatal periods suggests that, while the method may be satisfactorily used at any point in lung development, the correlation of radial count with age, body weight, body length, and other morphometric parameters is best in the intrauterine and early postnatal period. The method therefore seems particularly suited to evaluation of abnormalities of lung growth in the early phases of development.

We are grateful to Drs JL Paul and FP Glick for their invaluable statistical advice. Cynthia Sturgeon prepared the manuscript. The work was carried out with the support of March of Dimes grant 6-292 and Medical Research Council of Canada grant MT 7124.

\section{References}

${ }^{1}$ Emery JL, Mithal A. The number of alveoli in the terminal respiratory unit of man during late intrauterine life and childhood. Arch Dis Child 1960;35:544-7.

${ }^{2}$ Langston C, Thurlbeck WM. Lung growth and development in late gestation and early postnatal life. In: Rosenberg HS, ed. Perspectives in pediatric pathology. Vol 7. Chicago: Year Book Medical Publishers (in press).

${ }^{3}$ Langston C, Waszkiewicz E, Thurlbeck WM. A simple method for the representative sampling of lungs of diverse size. Thorax 1979;34:527-30. 